\section{Waardenburg syndrome with isolated deficiency of myenteric ganglion cells at the sigmoid colon and rectum}

\author{
Shun Watanabe, ${ }^{1}$ Shotaro Matsudera, \\ Takeshi Yamaguchi, ${ }^{1}$ Yukiko Tani, ${ }^{1}$ \\ Kei Ogino,1 Masanobu Nakajima, ${ }^{1}$ \\ Satoru Yamaguchi, ${ }^{1}$ Kinro Sasaki, ${ }^{1}$ \\ Hiroshi Suzumura,2 Takashi Tsuchioka ${ }^{1}$ \\ 1First Department of Surgery, \\ 2Department of Pediatrics, Dokkyo \\ Medical University, Japan
}

\begin{abstract}
Waardenburg syndrome (WS) has the characteristic clinical features caused by the embryologic abnormality of neural crest cells. WS patients sometimes suffer from functional intestinal obstruction. When it is Hirschsprung disease (HD), the WS is diagnosed as type 4 WS. We report a case of WS which did not have myenteric ganglion cells in the sigmoid colon and rectum. Whether to diagnosis this case as type 1 or $4 \mathrm{WS}$ is controversial. Moreover, this is the third report which has peristalsis failure caused by abnormal myenteric plexus. In all three cases, the eosinophils had aggregated in the myenteric layer of the transition zone. During embryonic life, enteric ganglion cells migrate to the myenteric layer from the proximal to the distal side sequentially and, subsequently, to the submucosal layer through the circular muscle. Therefore, we hypothesize that myenteric ganglion cells that had already migrated were eliminated by an eosinophil-mediated mechanism in these three cases. We believe this report may be helpful to elucidate the pathogenesis of some types of HD.
\end{abstract}

\section{Introduction}

In 1951, Waardenburg, a Dutch ophthalmologist and geneticist, described a rare syndrome, eponymously named the Waardenburg syndrome (WS), with the characteristic clinical features of developmental anomalies of the eyelids, eyebrows, and nose root with pigmentary defects of the iris and head hair, accompanied by congenital deafness. ${ }^{1}$ Moreover, WS is classified into 4 types. WS patients sometimes suffer from functional intestinal obstruction. When it is Hirschsprung disease (HD), the WS is diagnosed as type 4 WS.2,3

HD presents functional intestinal obstruction due to the congenital absence of ganglion cells in the distal colon. The cause of this disease is fairly well understood. The distal edges of the myenteric and submucosal ganglion cells are generally similar in HD. ${ }^{4}$

We report a case of WS which did not have myenteric ganglion cells in the sigmoid colon and rectum. To the best of our knowledge, there have only been two reports of peristalsis failure caused by abnormal myenteric plexus thus far. 5,6

\section{Case Report}

A 2-day-old female infant was admitted to our Neonatal Intensive Care Unit presenting with vomiting and abdominal distension. Her gestational age was 38 weeks. Her Apgar score was 9 and 10 at 1 and 5 min after birth, respectively. She weighed $2800 \mathrm{~g}$ at birth.

Her vital signs were within normal limits for her age at admission. Her abdomen was distended but soft. An abdominal radiograph at admission showed dilated intestines. The lower gastrointestinal series showed a caliber change at the sigmoid colon.

On day of life 8 , she had meconiumrelated ileus at the distal ileum. Therefore, we simultaneously performed an appendectomy and ileostomy and obtained an ileum biopsy. We didn't have reliable evidence other than caliber change which suggest she has Hirschsprung disease. Therefore, multiple biopsies for 8-day-old girl were too risky as lack of evidence at the time. The specimens of her appendix and ileum showed normal ganglia in both submucosal and myenteric plexus.

She didn't have rectoanal inhibitory reflex. In addition, her rectal biopsy showed acetylcholinesterase stain-positive nerve fibers in the submucosal layer. Therefore, we diagnosed her condition as HD.

Besides these findings, she had white skin all over her whole body, right eyelid droop, bilateral defects of the iridial pigments, and congenital bilateral sensorineural deafness (Figure 1A). Furthermore, a direct sequence revealed deletion of the SRY-related HMG-box 10 (SOX 10) gene. Therefore, we diagnosed her as type 4 WS tentatively.

When she was 5 months old, we performed an open-step biopsy from the ascending colon to the rectum to define the range of lesions. As with the lower gastrointestinal series, there was a caliber change at the sigmoid colon. The proximal intestine from the descending colon was normal size. Astonishingly, the specimens of sigmoid colon and rectum showed isolated deficien-
Correspondence: Shun Watanabe, First Department of Surgery, Dokkyo Medical University, 880 Kitakobayashi, Mibu-machi, Shimotsuga-gun, Tochigi 321-0293, Japan.

Tel.: +81.0282.86.1111 - Fax: +81.0282.86.6213. E-mail: shun-wat@dokkyomed.ac.jp

Key words: Waardenburg syndrome, Hirschsprung disease, Eosinophilic ganglionitis, Pathology.

Acknowledgments: The authors would like to thank T. Taguchi and K. Yoshimaru for providing assistance with pathological opinions.

Contributions: the authors contributed equally.

Conflict of interest: the authors declare no potential conflict of interest.

Funding: none.

Conference presentation: Part of this paper was presented at the 54th Annual Congress of the Japanese Society of Pediatric Surgeons, May 1113, 2017, Sendai, Japan.

Received for publication: 17 November 2017. Revision received: 13 March 2017.

Accepted for publication: 21 March 2017

This work is licensed under a Creative Commons Attribution NonCommercial 4.0 License (CC BY-NC 4.0).

(C) Copyright S. Watanabe et al., 2018

Licensee PAGEPress, Italy

Pediatric Reports 2018; 10:7500

doi:10.4081/pr.2018.7500

cies of myenteric ganglion cells with normal submucosal plexuses. Furthermore, the specimen of sigmoid colon showed eosinophilic aggregations in the myenteric layer (Figure 1B)

Though the pathological findings were specific, we determined her treatment plan as per recto-sigmoid type HD. Therefore, we planned an open Soave procedure when she was 1 year and 1 month old. We performed intraoperative pathological diagnoses. We transected the rectum and separated the mucosa from reversed anal canal. Subsequently, we performed a pull-through of the descending colon which has normal ganglia into the anal muscle barrel.

The resected specimen also showed isolated deficiency of myenteric ganglion in the distal side from the caliber change. There was no sign of eosinophil aggregation anywhere in the specimen (Figures 2 and 3 ).

The patient eventually established the self-evacuation exclusive of glycerin ene- 
mas and was discharged from treatment at 1 year and 5 months old.

She is 2 years and 6 months old. We are following up her and irrigating her colon once a month. Additionally, we are prescribing only probiotics. She has the selfevacuation exclusive of twice glycerin enema every day. Her height is on -2 SD and weight is on -1 SD. After she got a cochlear implant in right ear, her mental development is catching up with the normal level gradually.

\section{Discussion}

WS is a syndrome with the characteristic clinical features caused by a neural crest disorder. According to the diagnostic criteria for type $1 \mathrm{WS}^{3}$, the patient must have either (a) at least two of the major criteria or (b) one of the major criteria and two of the minor criteria. The major criteria are (I) sensorineural deficit hearing threshold, (II)
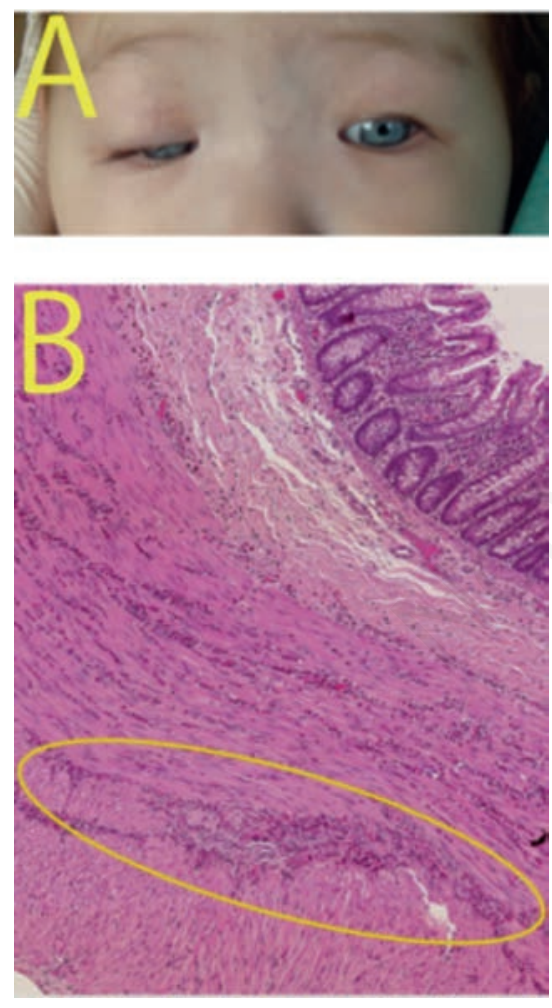

Figure 1. The patient's characteristic findings before open Soave procedure. A) The patient's facial features. She has bilateral defects of iridial pigment. B) The pathological biopsy findings at the sigmoid colon on 5 months old (the circle is the point of eosinophil aggregation). The eosinophils had aggregated in the myenteric layer (Hematoxylin \& eosin stain, magnification: $10 \times$ ). iris pigmentary abnormality, (III) hair hypopigmentation, (IV) dystopia canthorum, (V) first-degree relative previously diagnosed with WS. The minor criteria are (i) congenital leukoderma, (ii) synophrys or medical eyebrow flare, (iii) broad high nasal root, (iv) hypoplasia of alae nasi, (v) premature graying of the hair. Approximately $45-55 \%$ of WS are due to mutations within the gene encoding the
SOX10 transcription factor. ${ }^{7}$ A SOX 10 mutation is now considered an important cause of neurocristopathies including WS. 8

WS is classified into 4 types. Type 4 WS, also known as Shah-Waardenburg syndrome or Waardenburg-Hirschsprung disease, has the combined features of type 1 WS and HD (there were initially also notations of the megacolon instead of HD). ${ }^{7,9} \mathrm{~A}$ few WS patients suffer from functional
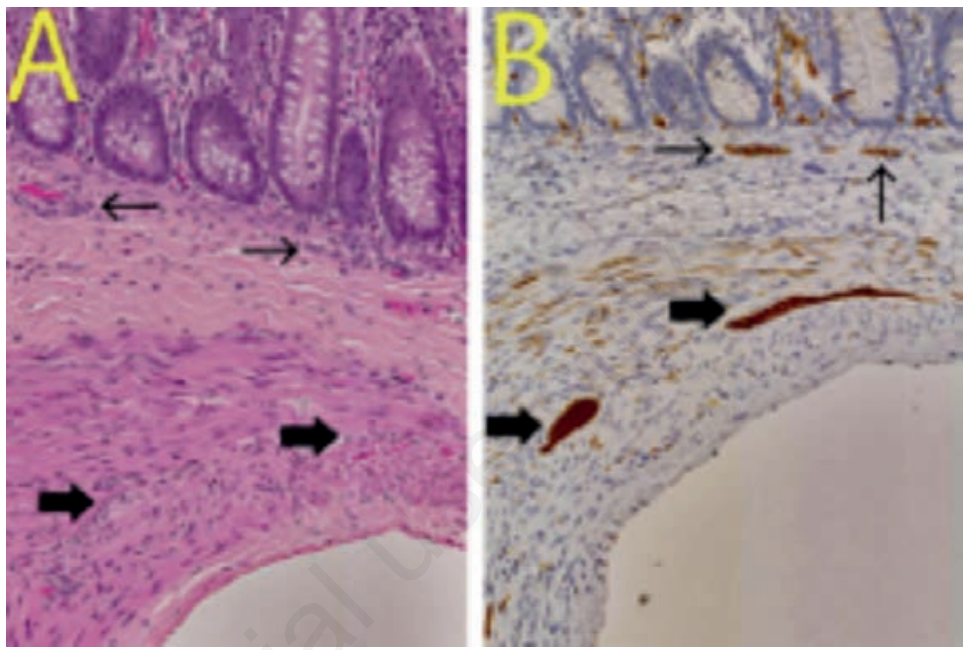

Figure 2. A-B) The pathological findings of resected specimen at the proximal sigmoid colon (the thin arrows are submucosal ganglion cells, and the thick arrows are myenteric ganglion cells). There are both submucosal and myenteric ganglion cells (A: Hematoxylin \& eosin stain, magnification: $4 \times$. B: Synaptophysin stain, magnification: $4 \times$ ).
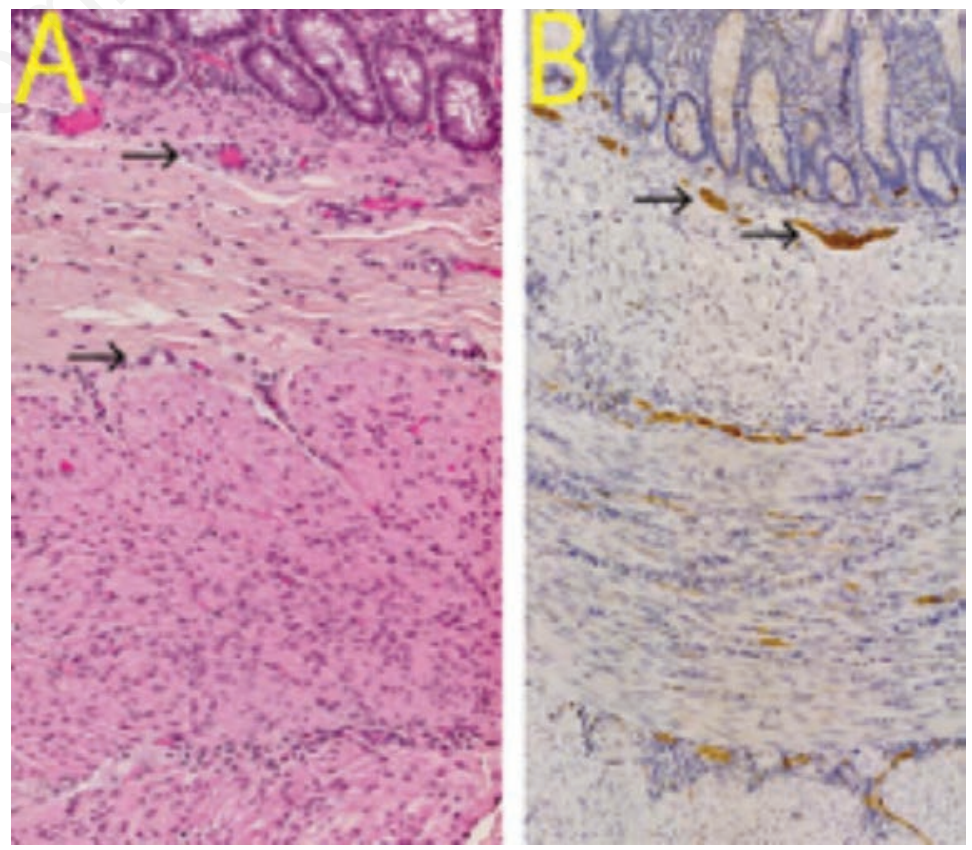

Figure 3. A-B) The pathological findings of the resected specimen at the rectum (the thin arrows are submucosal ganglion cells). The myenteric plexus does not have ganglion cells. Eosinophil aggregation is not found (A: Hematoxylin \& eosin stain, magnification: $4 \times . \mathrm{B}$ : Synaptophysin stain, magnification: $4 \times$ ). 
intestinal obstruction but without any aganglionosis. ${ }^{2}$ However, type 4 WS does not have definite diagnostic criteria (especially as to how far is the disease condition included in HD). ${ }^{3}$

HD is defined as a functional intestinal obstruction due to the congenital absence of ganglion cells in both Meissner and Auerbach plexuses in the distal colon. ${ }^{4,10}$ As is common in $\mathrm{HD}$, the distal edges of myenteric and submucosal ganglion cells are aligned. ${ }^{4}$ However, Kapur reported that the distal edge of submucosal ganglion cells was significantly more proximal than the distal edge of myenteric ganglion cells in 19/59 cases. ${ }^{11}$ The reverse was rare (1/59 cases) and the gap was small. In our case, the gap was too long.

Variant HD, sometimes called allied disorders of HD or pseudo-HD, is defined as a functional intestinal obstruction despite the presence of ganglion cells in both Meissner and Auerbach plexuses in the distal colon. ${ }^{12-14} \mathrm{HD}$ has abnormal ganglion cells in number, distribution or function and typically classified several types [immaturity of ganglia hypoganglionosis, intestinal neuronal dysplasia (IND), megacystis microcolon intestinal hypoperistalasis syndrome (MMIHS), segmental dilatation, internal anal sphincter achalasia and chronic intestinal pseudo-obstruction (CIP)].15,16

In functional intestinal obstruction, clinical feature and findings in this case (presence of caliber change, absence of rectoanal inhibitory reflex, acetylcholinesterase stain-positive nerve fibers in the submucosal layer) is similar to Hirschsprung disease and variant Hirschsprung disease. However, this case showed isolated deficiency of myenteric ganglion cells at the sigmoid colon and rectum. Strictly speaking, this type of pathology is different from HD or variant HD. Because our case fulfils (I) and (II) and showed deletion of the SOX 10 gene, we can diagnose her as type $1 \mathrm{WS}$ at least. However, whether to diagnose our case as type 1 or type 4 is unclear.

To the best of our knowledge, there have been only two reports of peristalsis failure caused by abnormal myenteric plexus.5,6 However, they are not WS. Remarkably, as with our case, the eosinophils had aggregated in the myenteric layer of the transition zone in both cases.

Kapur's report suggested eosinophilic ganglionitis at the transition zone in 18/60 cases. ${ }^{11}$ Furthermore, younger patients tended to show this finding more often than older ones. This is consistent with the disappearance of eosinophil aggregation in the resected specimen of our case.

In our patient, the eosinophils appeared in her peripheral blood at age 3 days. The count increased and reached a peak $(2700 / \mu \mathrm{L})$ at age 36 days. Around 9 months old, it decreased to within the normal range.

Although Lowichik and Weinberg first reported eosinophilic ganglionitis in 1997, ${ }^{17}$ its cause in HD is even less clear. They speculated that it was a secondary phenomenon because a correlation has not been observed between the severity of pathological ganglionitis and the clinical outcome.

The eosinophil inflammation of myenteric ganglia has been reported in some patients with variant HD. Moreover, immunosuppressive therapy is reportedly effective in rare cases of functional intestinal obstruction with eosinophilic myenteric ganglionitis. ${ }^{18}$

During embryonic life, enteric ganglion cells migrate sequentially in the myenteric layer from the proximal to the distal side and subsequently, to the submucosal layer through the circular muscle. ${ }^{19,20}$ Regarding one of the previously reported cases, ${ }^{6}$ Moore suggested that the isolated deficiency of myenteric ganglion cells seen therein could represent a secondary mechanism or apoptosis of the ganglia due to an immune reaction. ${ }^{4}$ We suggest that myenteric ganglion cells that had already migrated were eliminated by an eosinophil-mediated mechanism in these three cases.

\section{Conclusions}

In this case report, we share our experience of treating a pediatric patient with WS who did not have myenteric ganglion cells in the sigmoid colon and rectum. Based on the previous two reports and this case, we hypothesize that myenteric ganglion cells were eliminated by an eosinophil-mediated mechanism. We believe this report will help to understand and elucidate the pathogenesis of some types of HD.

\section{References}

1. Waadenburg PJ. A new syndrome combining developmental anomalies of the eyelids, eyebrows and nose root with pigmentary defects of the iris and head hair and with congenital deafness. Am J Hum Genet 1951;3:195-253.

2. Pingault V, Ente D, Dastot-Le Moal F, et al. Review and update of mutations causing Waardenburg syndrome. Hum Mutat 2010;31:391-406.

3. Farrer LA, Grundfast KM, Amos J, et al. Waardenburg syndrome (WS) type I is caused by defects at multiple loci, one of which is near ALPP on chromosome 2: first report of the WS consortium. Am J Hum Genet 1992;50:902-13.

4. Moore SW. Advances in understanding functional variations in the Hirschsprung disease spectrum (variant Hirschsprung disease). Pediatr Surg Int 2017;33:285-98.

5. Ariel I, Hershlag A, Lernau OZ, et al. Hypoganglionosis of the myenteric plexus with normal Meissner's plexus: a new variant of colonic ganglion cell disorders. J Pediatr Surg 1985;20:90-2.

6. Mishalany H, Olson A, Khan F, Santos A. Deficient neurogenic innervation of the myenteric plexus with normal submucous plexus involving the entire small and large bowel. J Pediatr Surg 1989;24:83-6.

7. Pingault V, Ente D, Dastot-Le Moal F, et al. Review and update of mutations causing Waardenburg syndrome. Hum Mutat 2010;31:391-406.

8. Chan KK, Wong CK, Lui VC, et al. Analysis of SOX10 mutations identified in Waardenburg-Hirschsprung patients: Differential effects on target gene regulation. J Cell Biochem 2003; 90:573-85.

9. Amiel J, Sproat-Emison E, GarciaBarcelo M, et al. Hirschsprung disease, associated syndromes and genetics: a review. J Med Genet 2008;45:1-14.

10. Whitehouse FR, Kernohan JW. Myenteric plexus in congenital megacolon: study of 11 cases. Arch Intern Med 1948;82:75-111.

11. Kapur RP. Histology of the transition zone in Hirschsprung disease. Am J Surg Pathol 2016;40:1637-46.

12. Puri P, Gosemann JH. Variant of Hirschsprung disease. Semin Pediatr Surg 2012;21:310-8.

13. Friedmacher F, Puri P. Classification and diagnostic criteria of variants of Hirschsprung's disease. Pediatr Surg Int 2013;29:855-72.

14. Obermayr F, Hotta R, Enomoto H, Young HM. Development and developmental disorder of the enteric nervous system. Nat Rev Gastroenterol Hepatol 2013;10:43-57.

15. Tomita R, Munakata K, Howard ER, Fujisaki S. Histological studies on Hirschsprung's disease and its allied disorders in childhood. Hepatogastroenterology 2004;51:10424.

16. Dingemann J, Puri P. Isolated hypoganglionosis:systemic review of a rare intestinal innervations defect. Pediatr Surg Int 2010;26:1111-5.

17. Lowichik A, Weinberg AG. Eosinophilic infiltration of the enteric 
neural plexus in Hirschsprung's disease. Ped Pathol Lab Med 1997; 17:885-91.

18. Schappi MG, Smith VV, Milla PJ, Lindley KJ. Eosinophilic myenteric ganglionitis is associated with function- al intestinal obstruction. Gut 2003;52:752-5.

19. McKeown SJ, Chow CW, Young HM. Development of the submucous plexus in the large intestine of the mouse. Cell
Tissue Res 2001;303:301-5.

20. Young HM, Newgreen D. Enteric neural crest-derived cells: origin, identification, migration, and differentiation. Anat Rec 2001;262:1-15. 